\title{
Screening for autism in preterm children with extremely low and very low birth weight
}

\author{
This article was published in the following Dove Press journal: \\ Neuropsychiatric Disease and Treatment \\ II February 2014 \\ Number of times this article has been viewed
}

\author{
Iva Dudova' \\ Martina Kasparova ${ }^{2}$ \\ Daniela Markova ${ }^{3}$ \\ Jana Zemankova ${ }^{4}$ \\ Stepanka Beranova' \\ Tomas Urbanek ${ }^{5}$ \\ Michal Hrdlicka' \\ 'Department of Child Psychiatry, \\ Charles University Second Faculty \\ of Medicine and University Hospital \\ Motol, Prague, Czech Republic; \\ ${ }^{2}$ Department of Pediatrics, Charles \\ University Second Faculty of Medicine \\ and University Hospital Motol, Prague, \\ Czech Republic; ${ }^{3}$ Department of \\ Pediatrics and Adolescent Medicine, \\ Charles University First Faculty of \\ Medicine and General University \\ Hospital, Prague, Czech Republic; \\ ${ }^{4}$ Department of Pediatrics, Charles \\ University Faculty of Medicine \\ and University Hospital, Hradec \\ Kralove, Czech Republic; Institute \\ of Psychology, Academy of Sciences, \\ Brno, Czech Republic
}

Correspondence: Iva Dudova Department of Child Psychiatry, Charles University Second Faculty of Medicine, University Hospital Motol, V Uvalu 84, I 5006 Prague, Czech Republic

Tel +4202 24433458

$\mathrm{Fax}+420224433420$

Email iva.dudova@Ifmotol.cuni.cz
Background: Studies of children with very low birth weight (VLBW, 1,000-1,500 g) and extremely low birth weight (ELBW, less than 1,000 g) indicate that this population seems to be at increased risk of autism spectrum disorder (ASD).

Methods: Parents of 101 VLBW and ELBW children (age 2 years, corrected for prematurity) agreed to participate in the study and signed informed consents; however, parents of only 75 children (44 boys, 31 girls) completed the screening questionnaires. The screening battery included the Modified Checklist for Autism in Toddlers (M-CHAT), Communication and Symbolic Behavior Scales Developmental Profile Infant-Toddler Checklist (CSBS-DP-ITC), and the Infant/Toddler Sensory Profile (ITSP). Children with disabilities were excluded. All children who screened positive on any of the screening tools were subsequently invited for a detailed assessment.

Results: Thirty-two children (42.7\%) screened positive on at least one of the screening questionnaires. The screening tool with the most positive results was the CSBS-DP-ITC (26 positive screens), followed by the M-CHAT (19 positive screens) and the ITSP (11 positive screens). Of the 32 children who tested positive, 19 participated in the detailed follow-up assessment. A diagnosis of ASD was confirmed in eight of the 19 children. ASD prevalence, calculated from those 19 children and those with negative screening results ( 43 children), yielded a prevalence of $12.9 \%$ in the sample. The difference in frequency of positive screens between the tests was significant $(P=0.011)$. In pair comparisons, ITSP was found to be significantly less positive than CSBS-DP-ITC $(P=0.032)$. No significant differences were found between the M-CHAT and CSBS-DP-ITC or between the M-CHAT and ITSP.

Conclusion: The results strongly support the hypothesis of an increased prevalence of autism in children with a birth weight less than $1,500 \mathrm{~g}$.

Keywords: autism spectrum disorder, preterm children, screening, Autism Diagnostic Observation Schedule, prevalence

\section{Introduction}

There is emerging evidence suggesting that low birth weight and prematurity may be risk factors for autism spectrum disorders (ASD). Interest in the topic was sparked in 2002 with a study by Elgen et al. ${ }^{1}$ Since then, several studies focusing on the prevalence of ASD in preterm children have been published. Research methodologies used differed substantially between these studies. They differ in features such as inclusion criteria, age at examination, screening instruments used, and use of clinical validation of screening results. Five studies used birth weight as an inclusion criterion (one study $<1,000 \mathrm{~g}$, two studies $\leq 1,500 \mathrm{~g}$, and two studies $\leq 2,000 \mathrm{~g}$ ), while another four used gestation 
weeks as the inclusion criterion (two studies $<26$ weeks, one study $<27$ weeks, and one study $<28$ weeks).

The most frequently used screening instrument was the Modified Checklist for Autism in Toddlers (M-CHAT). ${ }^{2}$ Limperopoulos et $\mathrm{al}^{3}$ used the M-CHAT to examine 91 preterm infants (birth weight $\leq 1,500 \mathrm{~g}$, mean age at examination 22 months), and found that $25 \%$ of the children screened positive. Kuban et $\mathrm{al}^{4}$ used the M-CHAT on a sample of 988 preterm children ( $<28$ weeks' gestation, aged 23-28 months at examination). The sample included those with cerebral palsy, cognitive impairment, and vision and hearing impairment. The M-CHAT-positive rate was $21 \%$ in the whole sample but only $10 \%$ in children without impairments. In a similar study, the M-CHAT was used to screen 523 preterm children ( $<26$ weeks' gestational age, aged 2 years at examination). ${ }^{5}$ The prevalence of positive M-CHAT screens was $41 \%$ in total and $16.5 \%$ in those without disabilities.

Other screening instruments have been used in studies of older children and adolescents. Elgen et $\mathrm{al}^{1}$ used the Asperger Syndrome Diagnostic Interview in 130 children (age 11 years, birth weight $\leq 2,000 \mathrm{~g}$ ) and found the prevalence of Asperger syndrome to be $1 \%$ compared with 131 normal birthweight controls. Indredavik et $\mathrm{al}^{6}$ compared 56 very low birth weight (VLBW) adolescents (birth weight $\leq 1,500 \mathrm{~g}$ ) with 83 term controls (age 14 years at examination). Using the Autism Spectrum Screening Questionnaire, they found that 7\% of VLBW adolescents had symptoms of Asperger syndrome versus $0 \%$ of controls. Hack et $\mathrm{al}^{7}$ screened 219 preterm children (birth weight $<1,000 \mathrm{~g}$, age 8 years) and compared them with 176 term children having similar sex, age, and maternal sociodemographic status. The Parent Child Symptom Inventory was utilized, and eight subjects (3.65\%) with ASD were identified in the preterm group, compared with one child $(0.57 \%)$ in the control group.

The first study that validated screening results with a clinical diagnosis was performed by Johnson et al. ${ }^{8}$ They screened 219 preterm children ( $<26$ weeks of gestation, age 11 years) using the Social Communication Questionnaire and compared the results with those in 153 term children. An ASD diagnosis was confirmed using the Development and Well Being Assessment, a semistructured diagnostic interview. They found ASD in $8 \%$ of the preterm children compared with $0 \%$ in controls. However, the only study in which highly reliable diagnostic instruments, such as the Autism Diagnostic Interview-Revised ${ }^{9}$ or the Autism Diagnostic Observation Schedule (ADOS) $)^{10}$ were used, was one performed by PintoMartin et al. ${ }^{11}$ Preterm children (birth weight $<2,000 \mathrm{~g}$, age 16 years) were screened and then clinically examined at age
21 years. The percentage of those with ASD was calculated to be $5 \%$ of the regional preterm birth cohort.

Stephens et al ${ }^{12}$ emphasized the simultaneous use of several screening tools in the infant population. Five hundred and fifty-four infants born at $<26$ weeks' gestation were screened between 18 and 22 months using the Pervasive Developmental Disorder Screening Test, second edition Stage 2 (PDDST), the response to name, and response to joint attention from the ADOS. Infants with severe cerebral palsy, deafness, and blindness were excluded. Of these, $20 \%$ of children had more than one positive screen, $10 \%$ had a positive PDDST, $6 \%$ response to name, and $9 \%$ response to joint attention. Only $1 \%$ screened positive on all three screens.

The aim of this study was to screen preterm infants by combining the advantages of the last two cited studies, ie, multiple screening tests simultaneously (in our case three) followed by validation of the diagnosis using the "gold standard" instrument of ADOS + clinical evaluation. We assumed that such a combined procedure would improve the accuracy of autism detection, not only in the preterm population, but also in the general pediatric population.

\section{Materials and methods}

\section{Sample}

Children with VLBW and extremely low birth weight (ELBW, less than 1,000 g) were consecutively recruited from March 2012 to May 2013. Three centers for newborns and infants at risk were involved: the Department of Pediatrics, University Hospital Motol, Prague; the Department of Pediatrics and Adolescent Medicine, General University Hospital, Prague; and the Department of Pediatrics, University Hospital, Hradec Kralove. Children with substantial disabilities, such as cerebral palsy or major vision and/or hearing impairments were excluded. The study was approved by the ethics committees of all three participating hospitals. Families were informed about the research project during routine checkups that took place regularly at 2 years of age, corrected for prematurity. Parents who agreed to participate in the study signed informed consents.

Parents of 101 VLBW and ELBW children agreed to participate in the study. Of these, families of 75 children (74.2\%) completed the screening questionnaires and sent them to the Department of Child Psychiatry. The sample comprised 44 boys and 31 girls, age 2 years (corrected for prematurity).

\section{ASD screening tools}

The screening battery included the: M-CHAT, the Communication and Symbolic Behavior Scales Developmental 
Profile Infant-Toddler Checklist (CSBS-DP-ITC), and the Infant/Toddler Sensory Profile (ITSP).

The M-CHAT ${ }^{2}$ is a widely used simple parental screen that consists of 23 yes/no items. Optimal age for administration is 24 months. There are six critical questions and 17 noncritical questions. A failed screening is defined if the parent reports that the child failed on any two critical questions or any three questions overall. In this case, a follow-up interview, consisting of additional corresponding questions, is recommended. Adequate reliability (Cronbach's alpha $=0.85)$ as well as high sensitivity $(0.87)$ and specificity $(0.99)$ have been described. ${ }^{2}$ Kleinman et al ${ }^{13}$ found a positive predictive value of 0.36 for the initial screening that improved to 0.74 when combined with the follow-up interview.

The CSBS-DP-ITC ${ }^{14}$ is a general broadband screen that detects a wide range of disorders such as global developmental delay, general language delay, and autism between 6 and 24 months. This 24-item parent-report questionnaire quantifies infant abilities into three subdomains, ie, social and emotional communication, receptive and expressive speech, and symbolic behavior. There was high internal consistency (Cronbach's alpha coefficients ranging from 0.86 to 0.92 ). Sensitivity estimates ranged from 0.87 to 0.93 and specificity was found to be $0.75 .{ }^{14}$ The positive predictive value was estimated to be $0.75 . .^{15}$

The ITSP $^{16}$ is a 48 -item caregiver questionnaire that measures sensory modulation abilities in children aged 7 months to 36 months (a version for infants aged 0-6 months is also available). Parents rate the frequency of their child's behavior on a 5-point scale from 1 (almost always) to 5 (almost never). Items are grouped into five sensory sections, ie, auditory processing, visual processing, tactile processing, vestibular processing, and oral sensory processing. Scores are then grouped into four quadrant scores, ie, low registration, sensation seeking, sensory sensitivity, and sensation avoiding. Section scores and quadrant scores are interpreted relative to age norms. Reliabilities for the various composite scores range from 0.69 to 0.85 . Some studies of the ITSP in ASD populations have been performed; ${ }^{17,18}$ however, the ITSP is not generally used for screening, ${ }^{19}$ and our study may be the first to evaluate its use as screening tool. We also established a new criterion for a positive screening. Participants were considered to have screened positive if results were definitely abnormal (ie, results outside two standard deviations of population norms) on at least two scores involving section and/or quadrant scores.

\section{Procedure}

The parents of children with VLBW and ELBW (2 years of age, corrected for prematurity) completed the screening battery questionnaires and sent them to the Department of Child Psychiatry at Motol University Hospital.

All children who had screened positive on any of the screening tools were subsequently invited for a detailed follow-up assessment. The assessment involved testing using the $\mathrm{ADOS}^{10}$ and a clinical examination by two experienced child psychiatrists with expertise in autism. The concept of best estimate clinical diagnosis, by consensus of two experienced specialists, was used as a gold standard. ${ }^{20}$ In cases of disagreement between the ADOS diagnosis and best estimate clinical diagnosis, the latter was preferred. The International Classification of Diseases, Tenth Edition (ICD-10) was used for clinical diagnoses. ${ }^{21}$

\section{Data analysis}

Statistical analysis was performed using Statistical Package for the Social Sciences version 22.0 software (IBM Corporation, Armonk, NY, USA). Descriptive statistics for the samples were used. The Mann-Whitney $U$ test and chi-squared test were used for analyzing differences between subsamples with positive and negative screens and the Cochran's $Q$ test was used for analyzing differences in positivity among tests. More detailed pair comparisons between tests were performed using paired Wilcoxon sign tests for false discovery rate adjusted for multiple testing.

\section{Results \\ Sample characteristics}

Table 1 shows the family and child characteristics. Thirty-two children (42.7\%) screened positive on at least one of the screening questionnaires. Analysis of children with positive (on at least one of the screening questionnaires) and negative screens found no significant differences relative to mean birth weight $(1,030.7 \mathrm{~g}$ versus $1,034.8 \mathrm{~g} ; U=687$, $P=0.9957)$, gestational age (28.1 weeks versus 28.6 weeks; $U=780.5, P=0.3239)$, length of stay in hospital (72.0 days versus 65.8 days; $U=607, P=0.3882$ ), or use of corticoids ( $25.0 \%$ versus $\left.23.3 \% ; \chi^{2}=0.031, d f=1, P=0.861\right)$.

\section{Prevalence of ASD in the sample}

As mentioned above, 32 children (42.7\%) screened positive on at least one of the screening questionnaires. Parents of 19 of the 32 children agreed to the more comprehensive follow-up assessment. A diagnosis of ASD was indicated in nine children based on the ADOS, and was confirmed 
Table I Family and child characteristics of the preterm population (age 2 years)

\begin{tabular}{ll}
\hline & $\begin{array}{l}\text { Mean (SD) or } \\
\text { frequency (\%) }\end{array}$ \\
\hline Maternal & \\
Age at delivery (years) & $32.8(4.5)$ \\
Education & \\
- Elementary & $0(0 \%)$ \\
- High school & $43(57.3 \%)$ \\
- University & $31(41.3 \%)$ \\
- Missing data & $1(1.3 \%)$ \\
Paternal & \\
Age at delivery (years) & $34.6(4.77)$ \\
Education & \\
- Elementary & $0(0 \%)$ \\
- High school & $45(60 \%)$ \\
- University & $26(34.7 \%)$ \\
- Missing data & $4(5.3 \%)$ \\
Child & \\
Birth weight (grams) & $1,033.1(293.1)$ \\
Gestational age (weeks) & $28.4(2.8)$ \\
Stay in hospital (days) & $68.4(31.5)$ \\
Use of corticoids & $18(24 \%)$ \\
\hline
\end{tabular}

Abbreviation: SD, standard deviation.

in eight of the 19 children. Diagnoses based on the ICD-10 were childhood autism $(n=5)$ and atypical autism $(n=3)$. In one case (one of nine), there was disagreement between the ADOS diagnosis and the clinical diagnosis. ADOS indicated ASD but the best estimated clinical diagnosis was mild mental retardation. ASD prevalence calculated from those examined (19 children) and those with negative screening results (43 children), was estimated to be as high as $12.9 \%$ of the sample.

\section{Screening battery}

The screening tool with the most positive results was CSBS-DP-ITC (26 positive screens) followed by M-CHAT (19 positive screens). The ITSP had the fewest positives (eleven positive screens). For details, see Table 2. Table 3 shows patterns of agreement between the three screening

Table 2 Sensitivity of screening tools in the preterm population (age 2 years)

\begin{tabular}{llll}
\hline Screening tool & $\mathbf{n}$ & Positive screens & Negative screens \\
\hline M-CHAT & 75 & $19(25.3 \%)$ & $56(74.7 \%)$ \\
CSBS-DP-ITC & 75 & $26(34.7 \%)$ & $49(65.3 \%)$ \\
ITSP & 72 & $11(15.3 \%)$ & $61(84.7 \%)$ \\
\hline
\end{tabular}

Notes: Cochran's $Q$ test for all three screening tools: $Q=9.07, d f=2, P=0.0$ I I; paired Wilcoxon sign tests (false discovery rate adjusted for multiple testing) CSBS-DP-ITC versus ITSP $(P=0.032)$.

Abbreviations: $\mathrm{n}$, number of screened children; M-CHAT, Modified Checklist for Autism in Toddlers; CSBS-DP-ITC, Communication and Symbolic Behavior Scales Developmental Profile Infant-Toddler Checklist; ITSP, Infant/Toddler Sensory Profile.
Table 3 Patterns of agreement among screening tools in the preterm population (age 2 years)

\begin{tabular}{llll}
\hline M-CHAT & CSBS-DP-ITC & ITSP & $\mathbf{n}$ \\
\hline 0 & 0 & 0 & 41 \\
0 & 0 & 1 & 5 \\
0 & $\mathrm{I}$ & 0 & 5 \\
0 & $\mathrm{I}$ & $\mathrm{I}$ & 3 \\
$\mathrm{I}$ & 0 & 0 & 2 \\
$\mathrm{I}$ & $\mathrm{I}$ & 0 & 13 \\
$\mathrm{I}$ & $\mathrm{I}$ & $\mathrm{I}$ & 3 \\
\hline
\end{tabular}

Abbreviations: M-CHAT, Modified Checklist for Autism in Toddlers; CSBS-DPITC, Communication and Symbolic Behavior Scales Developmental Profile InfantToddler Checklist; ITSP, Infant/Toddler Sensory Profile; $n$, frequency of the pattern; 0 , negative screening results; I, positive screening results.

tools, along with the frequency of each pattern. The three tools substantially agreed in negative cases $(n=41)$ but not in positive cases. The greatest similarity in positive screens was seen on the M-CHAT and CSBS-DP-ITC $(n=13)$. The difference in positive results between the screening tests was significant $(Q=9.07, d f=2, P=0.011)$. In pair comparisons, using the paired Wilcoxon sign tests with false discovery rate adjusted for multiple testing, the ITSP was found to be significantly less positive than the CSBS-DP-ITC $(P=0.032)$. No significant differences were found between the M-CHAT and CSBS-DP-ITC or between the M-CHAT and ITSP.

\section{Discussion}

Recent studies on the prevalence of ASD among prematurely born children, that validated screening results with clinical examinations and/or diagnostic instruments, estimated the ASD prevalence as being between $3.65 \%$ and $8 \%{ }^{7,8,11}$ The current prevalence of ASD in the general population of school-aged children has been found to be $1 \% .{ }^{22}$ The discrepancies in prevalence appear to be fairly obvious. Prematurity seems to be a rather nonspecific risk factor compared with specific risk factors for autism that have been previously suggested, such as tuberous sclerosis, fragile $\mathrm{X}$ syndrome, and various rare genetic syndromes. ${ }^{23}$ Losh et al, ${ }^{24}$ in a samesex twin study, estimated that every $100 \mathrm{~g}$ increase in birth weight provided a $13 \%$ reduction in risk of ASD.

Biological vulnerability factors in preterm children seem to be evident. Additionally, there is developing evidence that psychological factors are also important. Smith et a ${ }^{25}$ presented evidence from a sample of 44 children born at $<26$ weeks' gestation. Magnetic resonance imaging revealed that the number of stressors to which an infant was exposed was directly associated with decreased frontal and parietal brain width, and altered diffusion measures and functional connectivity in the temporal lobes. Additionally, increased abnormalities 
in motor behavior were observed during neurobehavioral examinations. Based on this information, various attempts have been made to improve observation, care, and rehabilitation of preterm children. ${ }^{26,27}$

In our study, which did not include children with major disabilities, $42.7 \%$ of the children screened positive on at least one of the screening questionnaires. This number could only be compared roughly (because of different methodologies) with three other available studies performed using children of comparable age., ${ }^{4,5}, 12$ The only study that used also a screening battery instead of a single tool was one by Stephens et al. ${ }^{12}$ They found a markedly smaller percentage of positive screens, with only $20 \%$ of infants (excluding those with disabilities) having at least one positive screen. However, they used less common screening instruments, ie, the PDDST and two parts from the ADOS (the response to name and response to joint attention).

The other two studies used the M-CHAT screening tool and found positive M-CHAT results in nondisabled infants to be $10 \%{ }^{4}$ and $16.5 \% .^{5}$ Positive M-CHAT tests in our sample was higher at $25.3 \%$. To our knowledge, neither study using the M-CHAT for screening preterm children completed the recommended follow-up telephone interview, which could have compromised the accuracy of the results. ${ }^{28}$ In our study, we used a follow-up clinical evaluation instead of the recommended telephone interview; however, we think this substitution improved the accuracy of our results.

The estimated ASD prevalence in our sample, based on follow-up clinical evaluation, was $12.9 \%$ of the screened children. The ASD prevalence might have been higher if all the children with positive screens had undergone the clinical examination, as was planned. Unfortunately, the parents of $40.6 \%$ of the children who screened positive did not bring their children for follow-up examination, and represents one of the limitations of our study. Other studies that verified screening results by further clinical examination were carried out in substantially older populations; Johnson et $\mathrm{al}^{8}$ examined 11-year-old children and found the ASD prevalence to be $8 \%$, and Pinto-Martin et al, ${ }^{11}$ working with young adults aged 21 years found an ASD prevalence of 5\%. If we consider "recovery from autism", which is a topic currently under intense discussion, ${ }^{29,30}$ to be a possibility, then the prevalence of autism at different ages might not be fully comparable. Another limitation of our study was the lack of a structured examination of motor and cognitive functions in preterm children that could be statistically analyzed and presented. We relied on the clinical judgment of pediatricians and child neurologists only.
It is important for clinical practice that the M-CHAT and CSBS-DP-ITC have been shown in our study to be comparable relative to positive results. Our assessment of the screening potential of the ITSP led to skepticism. Although the ITSP can differentiate different sensory profiles between very preterm children and controls at 2 years of age, ${ }^{31}$ its usefulness in autism screening seems to be limited. Regarding positive results, the ITSP was significantly inferior to the CSBS-DP-ITC in our study (15.3\% versus $34.7 \%, P=0.032$ ). The low positive rate of the ITSP could be the result of the overly strict criterion that we established. The ITSP was also inferior to the M-CHAT but the difference was not statistically significant, which could be a consequence of our small sample size, which also represents a study limitation. On the other hand, comparing screening instruments in this special population with more than a 10 -fold increase in prevalence does not significantly handicap the data, given that a lowerorder number of probands is needed in order to obtain reliable results. In any case, the study continues, and data from a larger sample will be presented in the future.

\section{Conclusion}

The results of this study strongly support the hypothesis of an increased prevalence of autism in children with birth weights less than 1,500 g. Both the M-CHAT and CSBSDP-ITC screening tests seem to be useful for screening the preterm population. We also found that the simultaneous use of more than one screening test offers increased screening sensitivity.

\section{Acknowledgments}

This study was supported by the Ministry of Education, Youth and Sports, Czech Republic (research grant COST LD11028), the Ministry of Health, Czech Republic (conceptual development of research organization, University Hospital Motol, Prague, Czech Republic 00064203), and by the ESF (COST Action ESSEA BM1004). The authors would like to thank Thomas Secrest for his assistance with the English version of the manuscript.

\section{Disclosure}

The authors declare that they have no competing interests in this work.

\section{References}

1. Elgen I, Sommerfelt K, Markestad T. Population based, controlled study of behavioural problems and psychiatric disorders in low birthweight children at 11 years of age. Arch Dis Child Fetal Neonatal Ed. 2002;87 F128-F132. 
2. Robins DL, Fein D, Barton ML, Green A. The Modified Checklist for Autism in Toddlers: an initial study investigating the early detection of autism and pervasive developmental disorders. J Autism Dev Disord. 2001;31:131-144

3. Limperopoulos C, Bassan H, Sullivan NR, et al. Positive screening for autism in ex-preterm infants: prevalence and risk factors. Pediatrics. 2008;121:758-765.

4. Kuban KC, O'Shea M, Allred EN, Tager-Flusberg H, Goldstein DJ, Leviton A. Positive screening of the Modified Checklist for Autism in Toddlers (M-CHAT) in extremely low gestational age newborns. J Pediatr. 2009;154:535-540.

5. Moore T, Johnson S, Hennessy E, Marlow N. Screening for autism in extremely preterm infants: problems in interpretation. Dev Med Child Neurol. 2012;54:514-520.

6. Indredavik MS, Vik T, Heyerdahl S, Kulseng S, Fayers P, Brubakk AM. Psychiatric symptoms and disorders in adolescents with low birth weight. Arch Dis Child Fetal Neonatal Ed. 2004;89:F445-F450.

7. Hack M, Taylor HG, Schluchter M, Andreias L, Drotar D, Klein N. Behavioral outcomes of extremely low birth weight children at age 8 years. J Dev Behav Pediatr. 2009;30:122-130.

8. Johnson S, Hollis C, Kochhar P, Hennessy E, Wolke D, Marlow N. Autism spectrum disorders in extremely preterm children. J Pediatr. 2010;156:525-531.

9. Lord C, Rutter M, LeCouteur A. Autism Diagnostic Interview-Revised: a revised version of a diagnostic interview for caregivers of individuals with possible pervasive developmental disorders. JAutism Dev Disord. 1994;24:659-685.

10. Lord C, Risi S, Lambrecht L, et al. The autism diagnostic observation schedule-generic: a standard measure of social and communication deficits associated with the spectrum of autism. J Autism Dev Disord. 2000;30:205-223.

11. Pinto-Martin JA, Levy SE, Feldman JF, Lorenz JM, Paneth N, Whitaker AH. Prevalence of autism spectrum disorders in adolescents born weighing <2000 grams. Pediatrics. 2011;128:883-891.

12. Stephens BE, Bann CM, Watson VE, et al. Screening for autism spectrum disorders in extremely preterm infants. J Dev Behav Pediatr. 2012;33:535-541.

13. Kleinman JM, Robins DL, Ventola PE, et al. The Modified Checklist for Autism in Toddlers: a follow-up study investigating the early detection of autism spectrum disorders. J Autism Dev Disord. 2008;38: $827-839$.

14. Wetherby AM, Brosnan-Maddox S, Peace V, Newton L. Validation of the Infant-Toddler Checklist as a broadband screener for autism spectrum disorders from 9 to 24 months of age. Autism. 2008;12:487-511.

15. Pierce K, Carter C, Weinfeld M, et al. Detecting, studying, and treating autism early: the one-year well-baby check-up approach. J Pediatr. 2011;159:458-465.
16. Dunn W. Infant/Toddler Sensory Profile. User's Manual. San Antonio, TX, USA: The Psychological Corporation; 2002.

17. Ben-Sasson A, Cermak SA, Orsmond GI, et al. Extreme sensory modulation behaviors in toddlers with autism spectrum disorders. Am J Occup Ther. 2007;61:584-592.

18. Ben-Sasson A, Cermak SA, Orsmond GI, Tager-Flusberg H, Kadlec MB, Carter AS. Sensory clusters of toddlers with autism spectrum disorders: differences in affective symptoms. J Child Psychol Psychiatry. 2008;49:817-825.

19. Eeles AL, Spittle AJ, Anderson PJ, et al. Assessment of sensory processing in infants: a systematic review. Dev Med Child Neurol. 2013;55:314-326

20. Kim SH, Lord C. Combining information from multiple sources for the diagnosis of autism spectrum disorders for toddlers and young preschoolers from 12 to 47 months of age. J Child Psychol Psychiatry. 2012;53:143-151.

21. World Health Organization. International Classification of Diseases. 10th ed. Geneva, Switzerland: World Health Organization; 1992.

22. Baron-Cohen S, Scott FJ, Allison C, et al. Prevalence of autismspectrum conditions: UK school-based population study. $\mathrm{Br} J$ Psychiatry. 2009;194:500-509.

23. Abrahams BS, Geschwind DH. Advances in autism genetics: on the threshold of a new neurobiology. Nat Rev Genet. 2008;9:341-355.

24. Losh M, Esserman D, Anckarsater H, Sullivan PF, Lichtenstein P. Lower birth weight indicates higher risk of autistic traits in discordant twin pairs. Psychol Med. 2012;42:1091-1102.

25. Smith GC, Gutovich J, Smyser C, et al. Neonatal intensive care unit stress is associated with brain development in preterm infants. Ann Neurol. 2011;70:541-549.

26. Guzzetta A, D'Acunto MG, Carotenuto M, et al. The effects of preterm infant massage on brain electrical activity. Dev Med Child Neurol. 2011;53 Suppl 4:46-51.

27. Guzzetta A, Pizzardi A, Belmonti V, et al. Hand movements at 3 months predict later hemiplegia in term infants with neonatal cerebral infarction. Dev Med Child Neurol. 2010;52:767-772.

28. Yirmiya N, Charman T. The prodrome of autism: early behavioral and biological signs, regression, peri- and post-natal development and genetics. J Child Psychol Psychiatry. 2010;51:432-458.

29. Helt M, Kelley E, Kinsbourne M, et al. Can children with autism recover? If so, how? Neuropsychol Rev. 2008;18:339-366.

30. Hrdlicka M, Dudova I. Controversies in autism: is a broader model of social disorders needed? Child Adolesc Psychiatry Ment Health. 2013;7:9.

31. Eeles AL, Anderson PJ, Brown N, et al. Sensory profiles of children born $<30$ weeks' gestation at 2 years of age and their environmental and biological predictors. Early Hum Dev. 2013;89:727-732.
Neuropsychiatric Disease and Treatment

\section{Publish your work in this journal}

Neuropsychiatric Disease and Treatment is an international, peerreviewed journal of clinical therapeutics and pharmacology focusing on concise rapid reporting of clinical or pre-clinical studies on a range of neuropsychiatric and neurological disorders. This journal is indexed on PubMed Central, the 'PsycINFO' database and CAS.
Dovepress

The manuscript management system is completely online and includes a very quick and fair peer-review system, which is all easy to use. Visit http://www.dovepress.com/testimonials.php to read real quotes from published authors. 